patients had hip involvement (70\% bilaterally), and $21.5 \%$ of them needed total hip joint replacement (TJR), which were significantly more frequent compared to control group. Two exon 10 MEFV variants were found in $69.4 \%$, and most $(69.8 \%)$ had homozygous M694V. Hip involvement was more frequent in patients with two exon 10 variants $(p=0.036$; $O R=4.4)$ compared to those with one variant; and TJR was more frequent in those with homozygous M694V compared to other exon 10 variants $(p=0.001 ; O R=10)$. Radiographic sacroiliitis was less frequent in patients with homozygous M694V ( $p=0.019$; OR=5.48). HLA-B27 positivity was not associated with hip or axial involvement in patients with FMF+SpA.

Biologics were used in 60 patients (anti-TNF in 43, secukinumab in 1, and tocilizumab in 2). Anti-IL-1 drugs were used in 23 patients for refractory FMF. In 9 patients, anti-TNF and anti-IL-1 drugs were tried for refractory joint involvement: 5 switched to anti-TNFs from anti-IL-1, 4 patients switched to anti-IL-1 from antiTNFs. Biologic DMARD requirement was more frequent in patients with two exon 10 variants $(p=0.006 ; O R=7.4)$, especially in those with homozygous M694V $(p=0.006$; OR=7.6). Although anti-IL-1 usage did not differ among MEFV variants, anti-TNF was used more frequently in patients with homozygous M694V $(p=0.007 ; O R=7.2) . F M F+S p A$ patients had higher serum CRP and developed amyloidosis more frequently than those patients with $\mathrm{SpA}$.

Table 1. Comparison of clinical and laboratory findings between the patients with FMF+SpA and SpA control

\begin{tabular}{lccc}
\hline & FMF + SpA $(n=100)$ & SpA $(n=217)$ & P value \\
\hline Age (years)* & $43.3 \pm 12$ & $43.4 \pm 11$ & 0.6 \\
Sex (n, \%) & 48 & $104(47.9)$ & 0.99 \\
Male & 52 & $113(52.1)$ & \\
Female & $181.6 \pm 108$ & $180.2 \pm 112$ & 0.8 \\
Duration of SpA (monnths) & $25.1 \pm 11$ & $28.4 \pm 8$ & 0.008 \\
Age onset of SpA (years)* & $35 / 80(43.8)$ & $79 / 212(37.3)$ & 0.3 \\
Peripheral arthritis (n, \%) & $6 / 21(28.6)$ & $105 / 139(75.5)$ & $<0.001(\mathrm{OR}=18.9)$ \\
HLA-B27 positivity (n, \%) & $26.7 \pm 25^{\star *}$ & $18.96 \pm 29$ & 0.001 \\
CRP (mg/dL) & $39.7 \pm 27$ & $39.4 \pm 28$ & 0.8 \\
ESR (mm/hour) & $47 / 96(49)$ & $23 / 118(19.5)$ & $<0.001(\mathrm{OR}=20.9)$ \\
Hip involvement (n, \%) & $20 / 93(21.5)$ & $8 / 205(3.9)$ & $<0.001(\mathrm{OR}=23.3)$ \\
TJR (n, \%) & $52 / 81(64.2)$ & $164 / 199(82.4)$ & $0.001(\mathrm{OR}=10.8)$ \\
Fulfilling mNY criteria (n, \%) & 60 & $68 / 214(31.8)$ & $<0.001(\mathrm{OR}=22.5)$ \\
Biologic DMARD (n, \%) & 46 & $68 / 214(31.8)$ & $0.015(\mathrm{OR}=5.96)$ \\
Anti-TNF (n, \%) & 16 & $5 / 205(2.4)$ & $<0.001(\mathrm{OR}=19.3)$ \\
Amyloidosis (n, \%) & & &
\end{tabular}

${ }^{*}$ mean \pm standard deviation, ${ }^{*}$ during the attack-free period

Conclusion: In this group of FMF+SpA patients, hip involvement and need for TJR were more frequent and associated with penetrant MEFV variants rather than HLA-B27 positivity. These patients had higher inflammatory response and risk of developing amyloidosis, and they needed biologics more frequently compared to SpA group. More severe disease course in $\mathrm{FMF}+\mathrm{SpA}$ patients requires further attention and analysis in larger cohorts.

Disclosure of Interests: None declared

DOI: 10.1136/annrheumdis-2021-eular.716

\section{POS1340 MULTICENTER STUDY OF 71 PATIENTS WITH REFRACTORY UVEITIS RELATED TO IMMUNE- MEDIATED INFLAMMATORY DISEASES ON CERTOLIZUMAB PEGOL TREATMENT}

J. L. Martín-Varillas ${ }^{1}$, V. Calvo-Río ${ }^{2}$ L. Sanchez-Bilbao², I. González-Mazón², A. Adan ${ }^{3}$, I. Hernanz Rodríguez ${ }^{3}$, A. Gallego ${ }^{4}$, E. Beltrán ${ }^{5}$, S. Castro 6 , P. Fanlo ${ }^{7}$ A. García Martos ${ }^{8}$, I. Torre-Salaberri ${ }^{9}$, M. Cordero-Coma ${ }^{10}$, J. De Dios-Jiménez Aberásturi ${ }^{11}$, Á. García-Aparicio ${ }^{12}$, M. Hernández-Garfella ${ }^{13}$, A. SanchezAndrade $^{14}$, A. García-Valle ${ }^{15}$, O. Maiz ${ }^{16}$, R. Miguélez ${ }^{17}$, S. Rodríguez Montero ${ }^{18}$, A. Urruticoechea-Arana ${ }^{19}$, R. Veroz Gonzalez ${ }^{20}$, A. Conesa ${ }^{21}$, C. FernándezCarballido $^{22}$, V. Jovani ${ }^{23}$, O. Martínez González ${ }^{24}$, P. Moya ${ }^{25}$, S. RomeroYuste $^{26}$, P. Rubio Muñoz ${ }^{27}$, E. Peña Sainz-Pardo ${ }^{28}$, M. A. González-Gay ${ }^{2}$, J. L. Hernández ${ }^{2}$, R. Blanco ${ }^{2} .{ }^{1}$ Hospital Sierrallana, Rheumatology, Torrelavega, Spain; ${ }^{2} \mathrm{H}$. U. Marqués de Valdecilla, Rheumatology, Santander, Spain; ${ }^{3} \mathrm{H}$. Clinic, Ophthalmology, Barcelona, Spain; ${ }^{4}$ H.U. de Badajoz, Rheumatology, Badajoz, Spain; ${ }^{5} \mathrm{H}$. del Mar, Rheumatology, Barcelona, Spain; ${ }^{6} \mathrm{H}$. U. Joan XXIII, Rheumatology, Tarragona, Spain; ${ }^{7}$ C.H. de Navarra, Internal Medicine, Navarra, Spain; ${ }^{8} \mathrm{H}$. U. del Tajo, Rheumatology, Madrid, Spain; ${ }^{\circ} \mathrm{H}$. Basurto, Rheumatology, Bilbao, Spain; ${ }^{10}$ C.A.U. de León, Ophthalmology, León, Spain; ${ }^{11}$ H. U. de Álava, Rheumatology, Vitoria, Spain; ${ }^{12}$ C.H.U. de Toledo, Rheumatology, Toledo, Spain; ${ }^{13}$ H.G.U. de Valencia, Ophthalmology, Valencia, Spain; ${ }^{14}$ H. Lucus Augusti, Rheumatology, Lugo, Spain; ${ }^{15}$ C.A.U. de Palencia, Rheumatology, Palencia, Spain; ${ }^{16}$ H.U. Donostia, Rheumatology, San Sebastian, Spain; ${ }^{17}$ H. Móstoles, Rheumatology, Madrid, Spain; ${ }^{18}$ H.U.Virgen de Valme, Rheumatology, Sevilla, Spain; ${ }^{19} \mathrm{H}$. Can Misses, Rheumatology, Ibiza, Spain: ${ }^{20}$ H. Merida, Rheumatology, Mérida, Spain; ${ }^{21}$ H. G.U. de Castellón, Rheumatology, Castellón, Spain; ${ }^{22} \mathrm{H}$. San Juan, Rheumatology, Alicante, Spain; ${ }^{23}$ H.G.U. de Alicante, Rheumatology,
Alicante, Spain; ${ }^{24}$ H.C.U. de Salamanca, Rheumatology, Salamanca, Spain; ${ }^{25}$ H. Santa Creu i Sant Pau, Rheumatology, Barcelona, Spain; ${ }^{26}$ C.H.U. de Pontevedra, Rheumatology, Pontevedra, Spain; ${ }^{27} \mathrm{H}$. de l'Esperit Sant, Rheumatology, Barcelona, Spain; ${ }^{28}$ H.U. 12 de Octubre, Pediatrics, Madrid, Spain

Background: Prognosis of non-infectious refractory uveitis has improved markedly with biologic therapy (BT) (1-5). Most data are with monoclonal anti-TNF drugs, especially Adalimumab (ADA) and Infliximab (IFX). However, there is not enough evidence for the use of Certolizumab Pegol (CZP).

Objectives: To evaluate the efficacy and safety of CZP in refractory uveitis secondary to Immune-Mediated Inflammatory Diseases (IMID).

Methods: Multicenter study of 71 patients with uveitis due to IMID refractory to glucocorticoids and conventional immunosuppressants. Efficacy was assessed with the following ocular parameters: best corrected visual acuity (BCVA), anterior chamber cells, vitritis, macular thickness and presence of retinal vasculitis. These outcomes were compared between baseline, 1st week, 1st and 6th month, and 1st and 2nd year. Statistical analysis was performed with IBM SPSS Statistics v.23.

Results: 71 patients/100 affected eyes (29 men/42 women) with mean age of $40.0 \pm 11.3$ years were studied. Underlying IMIDs were: spondyloarthritis $(n=38)$, Behçet (10), psoriatic arthritis (8), Crohn disease (3), sarcoidosis (2), JIA (1), reactive arthritis (1), rheumatoid arthritis (1), relapsing polychondritis (1), TINU (1), pars planitis (1), Birdshot (1) and idiopathic uveitis (3). Uveitis pattern was anterior $(n=55)$, posterior (6), panuveitis (6) and intermediate (4).

Prior to CZP, patients had received: methotrexate (37), sulfasalazine (26), azathioprine (14), cyclosporine (10), leflunomide (3), mycophenolate mofetil (3) and cyclophosphamide (1). Previous BT was administered in $48(67.6 \%)$ patients, with a mean of $1.4 \pm 1.3$ drugs per patient as follows: ADA ( $n=56)$, IFX (27), golimumab (14), tocilizumab (5) and etanercept (3). Pregnancy was the reason for prescribing CZP in 19 patients. CZP was administered in monotherapy $(n=39)$ or combined with conventional immunosuppressants $(n=32)$.

After a mean follow-up of $27.1 \pm 21.1$ months, most of the ocular variables showed a rapid and significantly improvement (Table 1). A decrease in the median number [IQR] of flares of uveitis before and after CZP, (3 [1-4] vs. 0 [0-1], p<0.001) was observed. CZP was discontinued in 15 patients due to remission $(n=2)$, ocular insufficient response (2) and incomplete response of extraocular manifestations (11). No serious adverse events were reported.

Conclusion: CZP seems to be effective and safe in patients with refractory uveitis due to IMID.

\section{REFERENCES:}

[1] Martín-Varillas JL, et al. Ophthalmology 2018; 125:1444-1451. doi: 10.1016/j. ophtha.2018.02.020.

[2] Atienza-Mateo B, et al. Arthritis Rheumatol 2019; 71:2081-2089. doi: 10.1002/ art.41026.

[3] Santos-Gómez M, et al. Clin Exp Rheumatol 2016; 34(6 Suppl 102):S34S40. PMID: 27054359

[4] Vegas-Revenga N, et al. Am J Ophthalmol 2019; 200:85-94. doi: 10.1016/j. ajo.2018.12.019

[5] Calvo-Río V, et al. Clin Exp Rheumatol. 2014; 32 (4 Suppl 84):S54-7. PMID: 25005576

Table 1.

\begin{tabular}{|c|c|c|c|c|c|c|}
\hline & Baseline & $1^{\text {st }}$ week & $1^{\text {st }}$ Month & $6^{\text {th }}$ Month & $1^{\text {st }}$ year & $2^{\text {nd }}$ year \\
\hline BCVA $($ mean \pm SD) & $0.68 \pm 0.27$ & $0.72 \pm 0.27^{*}$ & * $0.79 \pm 0.25^{*}$ & $0.84 \pm 0.24^{*}$ & $0.85 \pm 0.25^{*}$ & $0.87 \pm 0.22^{*}$ \\
\hline $\begin{array}{l}\text { Improvement } \\
\text { in AC Cells, } \\
\mathrm{n}(\%) \text { Patients } \\
\text { with AC cells at } \\
\text { baseline }(n=48)\end{array}$ & - & $21(43.7)$ & $30(62.5)^{\star}$ & $41(85.4)^{\star}$ & $48(100)^{\star}$ & $48(100)^{\star}$ \\
\hline $\begin{array}{l}\text { Improvement in } \\
\text { Vitritis, } \mathrm{n}(\%)\end{array}$ & - & $3(23.1)$ & $8(61.5)^{*}$ & $11(84.6)^{\star}$ & $13(100)^{*}$ & $13(100)^{\star}$ \\
\hline \multicolumn{7}{|l|}{$\begin{array}{l}\text { Patients with vitri- } \\
\text { tis at baseline } \\
(n=13)\end{array}$} \\
\hline $\begin{array}{l}\text { OCT }(\mu) \\
\quad(\text { mean } \pm \text { SD })\end{array}$ & $292.5 \pm 47.7$ & $294 \pm 47.4$ & $286.7 \pm 41.9^{\star}$ & $274.7 \pm 38.7^{\star}$ & $272.8 \pm 38.9^{\star}$ & $266.31 \pm 36.2^{*}$ \\
\hline $\begin{array}{l}\text { Choroiditis; } \\
\text { affected eyes, } \\
\mathrm{n},(\%)\end{array}$ & $3(4.2)$ & $3(4.2)$ & $2(2.8)$ & $2(2.8)$ & $1(1.4)$ & $0(0)$ \\
\hline $\begin{array}{l}\text { Retinal Vasculitis; } \\
\text { affected eyes, } \\
\mathrm{n},(\%)\end{array}$ & $2(2.8)$ & $0(0)$ & $1(1.4)$ & $0(0)$ & $0(0)$ & $0(0)$ \\
\hline
\end{tabular}

Disclosure of Interests: None declared

DOI: 10.1136/annrheumdis-2021-eular.1233 